\title{
Search for a matching or mismatching letter pair
}

\author{
LESTER E. KRUEGER and RONALD G. SHAPIRO \\ Ohio State University, Columbus, Ohio
}

\begin{abstract}
The perceptual matching task was modified in order to increase the error rate and thus to reveal more clearly whether internal noise more often changes an objective match into a perceived mismatch than vice versa (noisy-operator theory). In Experiment 1, subjects searched for a "same" pair in a list of "different" pairs or for a "different" pair in a list of "same" pairs. As predicted, the target pair was overlooked or missed more often on "different" lists. False alarms, though, were not higher on "same" lists, owing apparently to rechecking, which also produced slower search through "different" lists. To disable the rechecking mechanism, rapid serial visual presentation (RSVP) was used in Experiments 2 and 3. As predicted, misses exceeded false alarms on "different" lists, whereas the reverse held on "same" lists, both at the physical level (Experiment 2) and at the name level (Experiment 3). Letter set size and repetition were varied in Experiments 1 and 2, and the results indicate that when letter pairs are presented in close temporal and spatial proximity, subjects are influenced by extraneous interpair comparisons in addition to relevant intrapair comparisons in deciding whether or not a target pair is present.
\end{abstract}

According to the noisy-operator theory (Krueger, 1978), internal noise frequently will make two identical letters appear to be different, but rarely will make two differing letters appear to be identical. As a result, subjects can confidently respond "same" as soon as a match is perceived, since the match could not likely have been produced by internal noise, but cannot do likewise when a mismatch is perceived, since the mismatch could have been produced by internal noise. Instead, subjects must recheck the mismatch before responding "different." Thus, the noisy-operator theory can account for the typical speed advantage of "same" letter pairs over "different' letter pairs.

The noisy-operator theory has implications for errors as well as response time (RT), and errors will be the main focus of the present study. Rechecking will not eliminate all spurious mismatches, so some false"different" responses ought to occur on "same", trials. Few if any false-"same" responses ought to occur on "different" trials, on the other hand, because rarely will internal noise make two differing letters look identical. Of course, motor confusions may produce errors on both types of trials, but the overall prediction is that more errors will be made on "same" trials than on "different" trials. This error prediction has been confirmed in some previous studies, especially when the two letters in a pair were

This study was supported in part by National Institute of Mental Health Grant MH 32295. The authors are grateful to Bryan Connell, Sehchang Hah, Ernest Hallford, Larry Hettinger, Robert Hilkert, and Nancy Morris for assistance in collecting the data. Requests for reprints should be sent to Lester E. Krueger, Ohio State University, Human Performance Center, 404-B West 17th Avenue, Columbus, Ohio 43210. presented simultaneously rather than sequentially (Krueger \& Shapiro, 1981a), but many studies have found no significant difference between "same" and "different" trials on errors.

The error effect ought to be quite small and difficult to detect, since rechecking ought to eliminate most spurious mismatches, and some studies simply had too few subjects and trials to detect a small error effect. The present study tested two modifications of the perceptual matching task that may be used to make error tendencies more evident. First, in Experiment 1, several letter pairs were presented on each trial, and the subject had to search for the pair whose type did not fit that of the rest of the.list (e.g., an odd "same" pair on a "different" list). This task, which was used by Norman (1972), ought to make error tendencies more manifest because presenting several pairs increases the opportunities to make an error. Second, Experiments 2 and 3 used rapid serial visual presentation (RSVP), with subjects being force-fed the pairs at too rapid a rate to allow full rechecking. Disabling the rechecking mechanism ought to reveal more clearly any tendency for internal noise to produce more spurious mismatches than spurious matches.

\section{EXPERIMENT 1}

In the search task, the subject had to respond "yes" as soon as a target pair was detected (i.e., an odd "same" pair in a "different" list, or an odd "different" pair in a "same" list). On "catch" trials, in which no target pair was present, the subject had to respond "no" after searching through the entire list. Norman did not use catch trials. Instead, he had subjects name the target pair when they found it, and, although errors (misses) averaged $12 \%$, he did not 
report them separately for "same" and "different" lists. Using catch or target-absent trials enabled us not only to simplify the response, so that the subject merely had to press a "yes" or "no" button, but also to measure false alarms as well as misses, and to examine performance on a pure list composed solely of "same" pairs or "different" pairs. On target-present ("yes") lists, in contrast, the target type differs from the background or list type, so both pair types ("same," "different") would contribute to the performance.

The noisy-operator theory makes the following error predictions for the present task. On "same" lists, there ought to be relatively many false alarms on "no" (or target-absent) trials, because internal noise often will make one or more of the matching background pairs appear to be a mismatch, but there ought to be relatively few misses on "yes" (or targetpresent) trials, because internal noise rarely will make the target "different" pair appear to be a match. The opposite prediction holds for "different" lists. There ought to be relatively few false alarms on targetabsent trials, because internal noise rarely will make any of the mismatching background pairs appear to be a match, but there ought to be relatively many misses on target-present trials, because internal noise often will make the target "same" pair appear to be a mismatch.

The noisy-operator theory makes the following search time (ST) predictions for the present task. On target-absent trials, search ought to be slower through "different" lists than "same" lists, since the "different" pairs will receive rechecking. On target-present trials, the target type must be considered as well as the list type, and the target "same" pair on "different" lists ought to be processed faster than the target "different" pair on "same" lists. Since most pairs on a "different" list will still receive rechecking, though, search ought to be somewhat slower on "different" lists than on "same" lists. The fact that the "yes" response may be made before the entire list has been searched should further attenuate the difference in ST between "same" and "different" lists on target-present trials.

In Experiment 1, as well as in Experiment 2, the likelihood of interpair letter repetition was varied in order to determine whether this extraneous factor would affect performance. Two basic conditions were used. In one, only two letters were used to construct pairs, so that two letters located in two different (adjacent or nonadjacent) pairs on the list would be as likely to match as to mismatch, and the probability of letter repetition in a given position across adjacent pairs would be $50 \%$. In the second condition, five letters were used to construct pairs, and a given letter could not be repeated in the same letter position (left, right) across adjacent pairs $(0 \%$ repetition). Thus, two letters drawn from two different pairs would be much more likely to mismatch than to match. If people are compulsive comparers, as some evidence indicates (Krueger \& Shapiro, 1981b), then the predominance of extraneous mismatches in the five-letter 0\%-repetition case ought to make it more difficult to detect a target "different" pair in a "same" list, but easier to detect a target "same" pair in a "different" list. The disparity signaled by a target "different" pair would add little to that coming from the extraneous mismatches, and thus might be easily overlooked or disregarded. If so, then performance ought to suffer on "same" lists, but be better on "different" lists, since the detection there of a target "same" pair would benefit from the relative absence of extraneous interpair matches.

\section{Method}

Equipment. Uppercase letters were presented as thin, illuminated lines at a $60-\mathrm{Hz}$ refresh rate and at $32-\mathrm{cd} / \mathrm{m}^{2}$ intensity on a greenishtint, fast-decay P31 phosphor (decay to $1 \%$ intensity at $.25 \mathrm{msec}$ after display offset) by an Imlac PDS-4 graphics computer, which also measured ST to an accuracy of $1 \mathrm{msec}$. Each subject sat alone in a dark room, with the head held fast in a chinrest located $70 \mathrm{~cm}$ from the display screen.

Stimulus materials. Each letter was $.29 \mathrm{~cm}$ wide and $.43 \mathrm{~cm}$ high, and $.19 \mathrm{~cm}$ separated the two letters in each row. The vertical separation between adjacent rows was $.29 \mathrm{~cm}$. Each display contained 12 rows and thus was $.77 \mathrm{~cm}(.63 \mathrm{deg})$ wide and $8.35 \mathrm{~cm}$ (6.83 deg) high.

There were 232 displays in all, or 116 for each of the two main conditions (five-letter 0\%-repetition, two-letter 50\%-repetition), ordered as follows: two practice blocks of 6 trials each, then four regular blocks of 26 trials each ( 2 practice trials, 24 regular trials). List type ("same," "different") alternated by blocks, and before each new block a 2 -sec message on the screen indicated that "now the other type is odd." Of the 24 regular trials in a block, 12 displays contained no odd target pair, and 12 displays contained a single odd target pair, which was located once on each of the 12 rows. Four different stimulus tapes were prepared, so that half of the subjects received the five-letter $0 \%$-repetition condition first and the other half received it second, and half of the subjects received a "same"-list block first and the other half received it second.

In the five-letter $0 \%$-repetition condition, letters were sampled randomly from the set $F, G, J, K$, and $L$, except that no letter could appear in the same position in two adjacent pairs. The set was chosen so that each letter would be quite distinct from all others. In the two-letter 50\%-repetition condition, letters were randomly sampled from a set of two letters, and there was no restriction on repetition. For each list, a new set of two letters was randomly selected from the five-letter set.

Procedure. Subjects were asked to search down the column of letter pairs as rapidly as possible, but not at the expense of accuracy, and to respond "yes" as soon as an odd pair was detected or "no" if none was present. They were cautioned not to backtrack, but to respond "no" immediately after examining the last pair in the list. Subjects were asked to find the odd pair in several typed lists before the session began in order to verify that they understood the task.

Half of the subjects pressed a left-hand button for "yes" and a right-hand button for "no," and the other half had the reverse hand assignment. Prior to each trial, a fixation mark appeared alone for $700 \mathrm{msec}$ just below where the top pair of letters would appear. Subjects were asked to focus their eyes on this point and thus be ready to search down the list. Feedback on the accuracy of the response was provided during the 1.8-sec interval between trials. Mean ST will be reported below only for correct trials.

Subjects. Twenty Ohio State University undergraduates served 
as subjects in order to receive credit in an introductory psychology course. Data from two additional subjects were excluded because their errors exceeded $20 \%$. All subjects had at least $20 / 30$ vision (corrected) as tested with a Snellen chart.

\section{Results}

Mean ST was significantly longer when the odd pair was absent (vs. present) $[F(1,19)=175.67, p<.001]$ and when the list type was "different" (vs. "same") $[F(1,19)=20.01, p<.001]$, but was not affected by letter set size and repetition (five-letter $0 \%$ vs. twoletter $50 \%)(F<1$; see Table 1). The increased ST for "different" (vs. "same") lists was more evident when the odd pair was absent $[F(1,19)=28.32, p<$ $.001]$ and in the two-letter $50 \%$-repetition case $[\mathrm{F}(1,19)$ $=10.03, \mathrm{p}<.01]$ and was most evident of all when both of the latter two conditions held $[F(1,19)=$ $8.52, \mathrm{p}<.01$; see Table 1]. Separate analyses indicated that search was significantly slower through "different" lists both for the five-letter 0\%-repetition trials considered alone $[F(1,19)=5.74, p<.05]$ and for the two-letter $50 \%$-repetition trials considered alone $[F(1,19)=26.62, p<.001]$.

Errors were significantly higher on "different" lists $[F(1,19)=10.49, p<.01]$, and misses on targetpresent trials significantly exceeded false alarms on target-absent trials $[F(1,19)=69.17, p<.001]$, but errors were not affected by letter set size and repetition (five-letter $0 \%$ vs. two-letter $50 \%$ ) $[\mathrm{F}(1,19)=$ 1.47 , n.s.; see Table 1]. The increase in errors on "different" (vs. "same") lists was more evident when the target pair was present (misses) $[F(1,19)$ $=5.39, \mathrm{p}<.05]$. Separate analyses indicated that the higher error rate for "different" lists was significant for the two-letter $50 \%$-repetition trials considered alone $[F(1,19)=19.66, \mathrm{p}<.001]$, but not for the five-letter $0 \%$-repetition trials considered alone $[F(1,19)=2.48$, n.s. $]$.

\section{Discussion}

The predictions of the noisy-operator theory were generally confirmed, both on ST and errors. It took

Table 1

Experiment 1: Mean Search Time (ST) in Milliseconds and Percentage of Error Rate (PE) by Target Absence vs. Presence, Letter Set Size, Letter Repetition (in Percent), and List Type

\begin{tabular}{|c|c|c|c|c|c|}
\hline \multirow{3}{*}{$\begin{array}{c}\text { Set } \\
\text { Size }\end{array}$} & \multirow{3}{*}{$\begin{array}{c}\text { Repeti- } \\
\text { tion }\end{array}$} & \multicolumn{4}{|c|}{ List Type } \\
\hline & & \multicolumn{2}{|c|}{ "Same" } & \multicolumn{2}{|c|}{ "Different" } \\
\hline & & ST & PE & ST & $\mathrm{PE}$ \\
\hline \multicolumn{6}{|c|}{ Target Present } \\
\hline $\begin{array}{l}2 \\
5\end{array}$ & $\begin{array}{r}50 \\
0\end{array}$ & $\begin{array}{l}2181 \\
2292\end{array}$ & $\begin{array}{r}10.42 \\
9.38\end{array}$ & $\begin{array}{l}2388 \\
2378\end{array}$ & $\begin{array}{l}17.50 \\
14.17\end{array}$ \\
\hline \multicolumn{6}{|c|}{ Target Absent } \\
\hline $\begin{array}{l}2 \\
5\end{array}$ & $\begin{array}{r}50 \\
0\end{array}$ & $\begin{array}{l}3116 \\
3474\end{array}$ & $\begin{array}{l}1.67 \\
2.08\end{array}$ & $\begin{array}{l}3925 \\
3805\end{array}$ & $\begin{array}{l}2.50 \\
2.08\end{array}$ \\
\hline
\end{tabular}

longer to search through a "different" list for a target "same" pair than through a "same" list for a target "different" pair. This effect was smaller when the target was present than when it was absent, probably both because a target "same" pair itself was processed faster than a target "different" pair and because search was less extensive (i.e., self-terminating) on target-present trials.

The search task fulfilled its promise of producing a high error rate, and, as predicted by the noisyoperator theory, there were more misses on "different" (vs. "same") lists, which accounted for the significantly higher error rate on "different" lists. Contrary to predictions, the reverse trend, that is, more false alarms on "same" (vs. "different") lists, did not materialize. Since the lists were open to view, subjects could and apparently did do additional rechecking when a potential target pair was detected, thereby eliminating virtually all incipient false alarms. The nearly uniform, $2 \%$ false-alarm rate found for all conditions (see Table 1) is about what would be expected based on motor confusions alone. Thus, ordinary rechecking slowed the search through "different" lists, and additional rechecking eliminated the false alarms. It will be interesting to observe what happens when rechecking is prevented in Experiments 2 and 3 by using RSVP.

Subjects in Experiment 1 were compulsive not only in making additional intrapair comparisons (rechecking), but also in making additional, extraneous interpair comparisons. The interpair comparisons would have produced an equal number of extraneous matches and mismatches in the two-letter 50\%-repetition condition, but many more extraneous mismatches than matches in the five-letter $0 \%$-repetition condition. The latter result would make it more difficult to detect a target "different" pair, but easier to detect a target "same" pair, and this can explain why performance in the five-letter $0 \%$-repetition condition was worse on "same" lists, but better on "different" lists, as compared with the two-letter 50\%-repetition condition. Both conditions produced data that generally confirmed the ST and error predictions of the noisy-operator theory, but the two-letter $50 \%$ repetition condition did so to a greater extent than the five-letter $0 \%$-repetition condition (see Table 1 ). The two-letter 50\%-repetition condition, it might be noted, provides a fairer means to test the noisyoperator theory. Not only does that condition equate the proportion of extraneous matches and mismatches, but, by using only two letters, it also equates the relative frequency of "same" and "different" pairs. When five letters are used, in contrast, there are fewer unique "same" pairs than unique "different" pairs.

It is interesting that the extraneous interpair matches and mismatches seemingly were fed directly into the same decision process as the relevant intrapair ones. 
Krueger and Shapiro (1981b) found that letter repetition from one pair to the next in a discrete-response task did not produce a tendency to respond "same," as the present results would predict, but rather a tendency to keep the same response as on the preceding pair or trial, whether "same" or "different." There was a considerable temporal interval between pairs in their case, since each pair received its own response, and that may have made it easier to filter out the extraneous interpair comparisons at the perceptual level, although they yet had an effect at the cognitive level (i.e., in deciding whether to keep or change the preceding response).

The seeming inability of our subjects to restrict their attention to intrapair comparisons can help to explain Norman's (1972) results. He found an even larger shift toward better performance on "different" lists than was evident here in the five-letter $0 \%$ repetition condition. In fact, search was $20 \%$ faster through "different" lists than "same" lists in his Experiment 4 . He made interpair comparisons easier to do and more difficult to ignore by packing his letter pairs much more closely together (his 48-pair list was $.63 \mathrm{deg}$ wide and $10.43 \mathrm{deg}$ high, whereas our 12pair list was $.63 \mathrm{deg}$ wide and $6.83 \mathrm{deg}$ high). In addition, he increased the preponderance of extraneous interpair mismatches over matches by using all letters except $\mathrm{I}$ and $\mathrm{J}$, and by eliminating immediate repetition across position as well as within position (i.e., no letter was repeated in successive pairs).

Information would be combined even more directly across rows if adjacent pairs formed integral rather than separable units. Krueger (1973) found that extraneous surrounding material (underlines, plus signs, equal signs) produced a tendency to respond "different" if it was asymmetrical (i.e., a different type or amount of material abutted each letter in a pair). It is conceivable, then, that the target "same" pairs on "different" lists often were misperceived as mismatching, not because of internal noise (noisy-operator theory), but because the "same" pair was integrated with adjacent "different" pairs. However, Krueger (1973) presented the extraneous material quite close to the letter pair or presented it and the letter pair alone in the visual field, both of which ought to have favored perceptual integration. In the present case, in contrast, 12 pairs were presented at one time, and approximately the same amount of material abutted each letter in a pair, so the adjacent rows could be readily segregated as separate units. Therefore, we dismiss the possibility that perceptual integration rather than internal noise led subjects to miss the target "same" pairs on "different" lists.

\section{EXPERIMENT 2}

Experiment 2 differed from Experiment 1 mainly in that subjects did not have full view of the display, but saw one letter pair at a time with RSVP. It was assumed that subjects could continue to process the preceding pair while a new pair was being shown (Fischler, 1975; Sternberg \& Scarborough, Note 1), but that a fast input rate would prevent rechecking of the encoding and comparison operations. If rechecking is eliminated, then, according to the noisyoperator theory, many false alarms but few misses ought to occur on "same" lists, since many of the background "same" pairs and typically the odd "different" pair itself will be perceived as mismatching. By way of contrast, many misses but few false alarms ought to occur on "different" lists, since few of the background "different" pairs and often not even the odd "same" pair itself will be perceived as matching. Eliminating rechecking also ought to reduce or eliminate the difference in speed of processing of different types of pairs, so we expected little, if any, RT effect. Krueger and Shapiro (1979), in studying the word-superiority effect, found that RSVP reduced or eliminated the usual RT effect, but increased the error effect. It was not clear what presentation rate would suffice to eliminate rechecking, but not the initial encoding and comparison as well, so a wide range of presentation rates was used in Experiment 2.

\section{Method}

The method was the same as in Experiment 1, except for the following changes. Only one letter pair was shown at a time, centered just above the fixation mark, which subjects were asked to focus on at all times. Prior to each trial, a fixation mark appeared alone for $500 \mathrm{msec}$; then 11 pairs were presented, one by one, in the same location. The presentation rate was $2,3,4$, or 5 pairs/sec. To let subjects adjust their monitoring appropriately and not be disrupted by sudden, unexpected changes in presentation rate, the rate was varied in a regular fashion. Starting at the slowest level, it gradually became faster until it reached the fastest level, then it declined. Two such cycles occurred within each main block.

Letter set size and repetition was a between-subjects variable rather than a within-subjects variable, as in Experiment 1 . In the five-letter $0 \%$-repetition condition, the items were presented continuously, with no break between pairs, so the exposure duration per pair was $500,333,250$, or $200 \mathrm{msec}$ at a presentation rate of $2,3,4$, or 5 pairs/sec, respectively. Letters were sampled randomly from the set $F, G, J, K$, and $L$, except that no letter could appear in the same position in two pairs in immediate succession. In the two-letter $50 \%$-repetition condition, the letters were sampled randomly from the set $J$ and $K$, and there were no constraints to prevent letter repetition. The exposure duration per pair was fixed at $100 \mathrm{msec}$ in order to prevent the fusion of repeated letters, and after each letter pair was shown the fixation mark appeared alone for $400,233,150$, or $100 \mathrm{msec}$ at a presentation rate of $2,3,4$, or 5 pairs/sec, respectively. Even at the fastest input rate (i.e., $5 / \mathrm{sec}$ ), there was a noticeable flicker between successive pairs when letters were repeated.

The target pair, when present, appeared as the third, sixth, or ninth pair in the 11-pair series. There were two regular blocks, one for "same" lists and the other for "different" lists, and half of the subjects received each list type first. Each block contained 96 regular trials, or four replications each of the following combinations of conditions: three target pair positions (Pairs 3, 6, 9 on "yes" trials; not present on "no" trials), two response types ("yes," "no"), and four presentation rates $(2,3,4,5$ pairs $/ \mathrm{sec})$ $(4 \times 3 \times 2 \times 4=96)$. Two practice trials preceded each block, and, in addition to the two regular blocks ( 98 trials each), there 
were two practice blocks ( 8 trials each), for a total of 212 trials. There were 40 subjects; 20 participated in the two-letter $50 \%$ repetition condition, and the other 20 participated in the fiveletter $0 \%$-repetition condition.

\section{Results}

The data from the two-letter 50\%-repetition condition are shown in Figure 1, and those from the fiveletter 0\%-repetition condition are shown in Figure 2. Target-present or "yes" RT was measured from the onset of the target pair in the 11-pair list, and targetabsent or "no" RT from the projected onset of the (nonexistent) 12th pair, or the presumed first instant in which the subject knew that the list was terminated. Mean RT was not significantly affected by letter set size and repetition $(F<1)$ or by presentation rate $(F<1)$, but was significantly shorter on targetabsent trials $[\mathrm{F}(1,36)=15.55, \mathrm{p}<.001]$ and on "different" lists $[F(1,36)=7.71, p<.01]$. The shorter RT on target-absent trials indicates that the failure of the 12th pair to appear was more salient than the appearance of a target pair earlier in the list, and this effect was more evident at slower rates of presentation $[F(3,108)=3.66, p<.025]$. The shorter $R T$ on "different" lists largely reflects the shorter RT required to respond to a target "same" pair in a "dif-

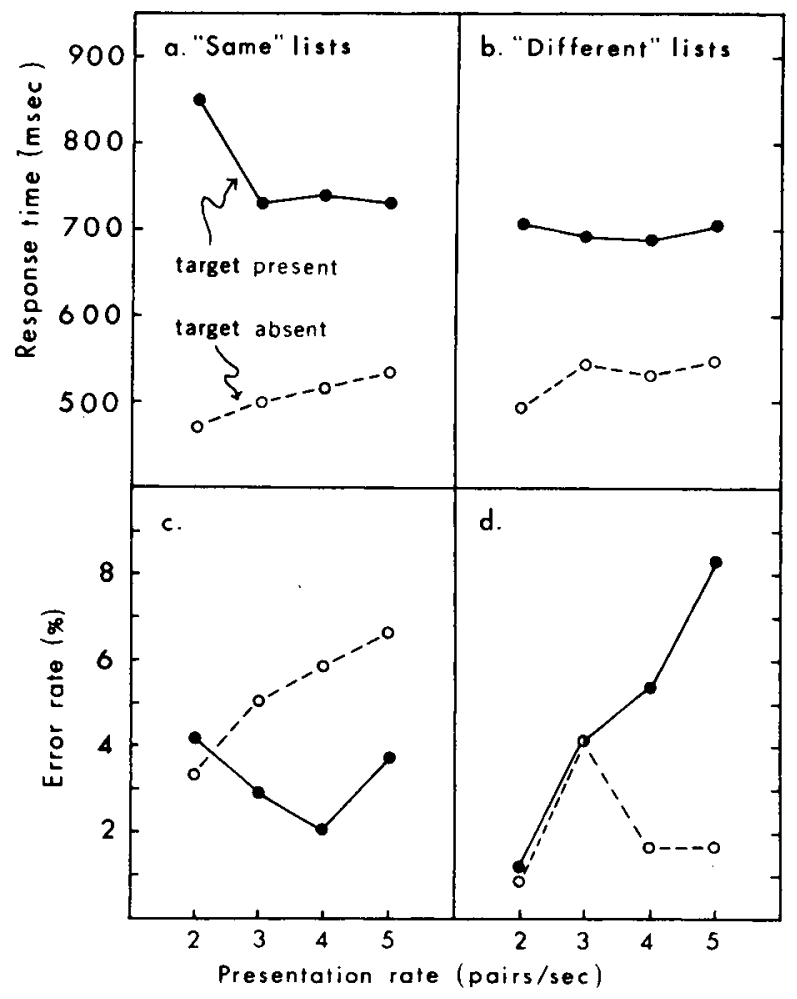

Figure 1. Experiment 2, two-letter 50\%-repetition condition. Mean RT and percent error rate by presentation rate, list type, and target presence vs. absence. (Target-present RT was measured from the onset of the target pair; target-absent $R T$ was measured from the projected onset of a 12th pair.)

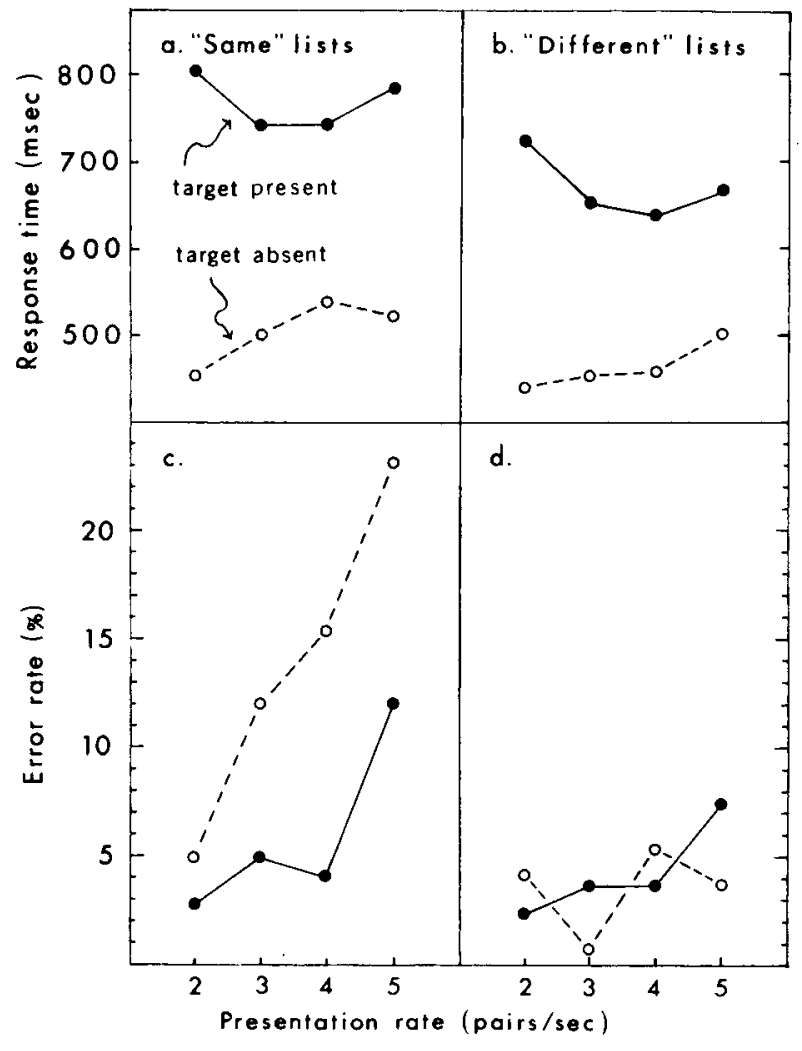

Figure 2. Experiment 2, five-letter 0\%-repetition condition. Mean RT and percent error rate by presentation rate, list type, and target presence vs. absence. (Target-present RT was measured from the onset of the target pair; target-absent $R T$ was measured from the projected onset of a 12th pair.)

ferent" list (vs. a target "different" pair in a "same" list), because, as Figures 1 and 2 reveal, this effect was much more evident on target-present (vs. targetabsent) trials $[F(1,36)=5.75, \mathrm{p}<.025]$.

Errors were significantly higher in the five-letter 0\%-repetition condition (Figure 2) than in the twoletter $50 \%$-repetition condition (Figure 1) $[F(1,36)=$ $11.20, \mathrm{p}<.01]$, on "same" (vs. "different") lists $[F(1,36)=28.35, p<.001]$, on target-absent (vs. target-present) trials $[F(1,36)=6.75, p<.025]$, and when the rate of presentation was high $[F(3,108)=$ $15.97, \mathrm{p}<.001]$. However, the latter three effects were restricted to the five-letter $0 \%$-repetition condition (see Figure 2), as indicated by significant interactions between letter set size and repetition and list type $[F(1,36)=16.82, p<.001]$, target presence $[F(1,36)=10.11, p<.01]$, and rate of presentation $[F(3,108)=4.93, p<.01]$. In separate analyses, none of the latter three variables had a significant effect on errors in the two-letter $50 \%$-repetition condition considered alone, whereas in the five-letter $0 \%$ repetition condition considered alone, errors were significantly higher on "same" lists $[F(1,18)=26.89$, $\mathrm{p}<.001]$, on target-absent trials $[\mathrm{F}(1,18)=11.44$, 
$\mathrm{p}<.01]$, and when the rate of presentation was high $[F(3,54)=16.61, \mathrm{p}<.001]$.

There was a significant interaction on errors between list type and rate of presentation $[\mathrm{F}(3,108)=$ $3.54, \mathrm{p}<.01]$, reflecting a larger increase in errors on "same" (vs. "different") lists as the rate increased. Significant higher order interactions indicated that this divergence occurred on false alarms (targetabsent trials), but not on misses (target-present trials) $[F(3,108)=5.49, p<.01]$, and that it occurred in the five-letter $0 \%$ letter repetition condition, but not in the two-letter $50 \%$ letter repetition condition $[F(3,108)$ $=7.99, \mathrm{p}<.001]$. In separate analyses, the list type $x$ rate interaction was significant in the five-letter $0 \%$-repetition condition considered alone $[F(3,54)=$ $8.07, \mathrm{p}<.001]$, but not in the two-letter $50 \%$-repetition condition considered alone.

As predicted by the noisy-operator theory, there also was a significant interaction on errors between list type and target presence $[F(1,36)=42.43, p<.001]$. False alarms predominated on "same" lists, and misses predominated on "different" lists. This pattern was more clearly evident in the two-letter $50 \%$ repetition condition (see Figure 1), but not significantly so, and in separate analyses the interaction was significant both in the five-letter $0 \%$-repetition condition considered alone $[\mathrm{F}(1,18)=25.85$, $\mathrm{p}<$ $.001]$ and in the two-letter $50 \%$-repetition condition considered alone $[F(1,18)=16.95, p<.001]$. Within the five-letter $0 \%$-repetition condition, the predominance of false alarms over misses on "same" lists was significant $[F(1,18)=30.15, p<.001]$, but not the slight reverse tendency (predominance of misses over false alarms) on "different" lists $(F<1)$. Within the two-letter 50\%-repetition condition, the predominance of false alarms on "same" lists was significant $[F(1,18)=5.02, p<.05]$, as was also the predominance of misses on "different" lists $[F(1,18)=10.96$, $\mathrm{p}<.01]$.

\section{Discussion}

The findings in Experiment 2 provide further confirmation of the error predictions of the noisy-operator theory. False alarms predominated on "same" lists, whereas misses predominated on "different" lists, as would be expected if "same" pairs were more likely to be misperceived as "different" than vice versa. As in earlier studies (Krueger \& Shapiro, 1979, 1980), using RSVP and presenting items one after the other in the same location made errors a more sensitive dependent variable than $\mathrm{RT}$, and it proved especially useful here in revealing the true tendency to make false alarms. The RSVP method thus ought to be especially useful when information on error effects is needed to interpret RT effects (Krueger, 1978; Krueger \& Shapiro, 1980).

In Experiment 2, subjects again let extraneous interpair comparisons affect their decisions. As in Ex- periment 1 , there was a marked shift toward better performance on "different" lists when most extraneous interpair comparisons signaled mismatch rather than match (see Figure 2; five-letter 0\%-repetition condition). It would be very difficult for a subject to distinguish the added effect of a lone odd "different" pair on a "same" list from that of the extraneous mismatches in this case, and the very high false-alarm rate on "same" lists (nearly $25 \%$ at the fastest input rate, as Figure 2 shows) indicates that subjects often mistook an extraneous interpair mismatch for a real intrapair mismatch (i.e., a target "different" pair).

As in Experiment 1, it is conceivable that letters sometimes were perceptually integrated or fused across pairs. If so, then the frequent misses of the target "same" pair on "different" lists could have been produced, not by interruption of rechecking, but by the integration of the "same" pair with the prior or succeeding "different" pairs. However, we favor the interruption, or stop-processing, model over the integration model in the present case. Each letter pair was presented long enough to be perceived as a distinct entity, even at the fastest rate, and the use of an 11-pair list made it even less likely that perceptual fusion would occur between any two adjacent pairs. The phenomenal impression in watching the RSVP display was that each pair was perceived as such before being replaced by the next pair. Some motion and streaming effects were evident in the twoletter 50\%-repetition condition, though, when a particular letter was repeated several times in a row, but these effects reflected, not the failure to distinguish successive pairs, owing to integration, but, rather, the ability to detect repetition between separate pairs. To eliminate these perceptual effects, name matches rather than physical matches were studied in Experiment 3.

\section{EXPERIMENT 3}

In Experiment 3, the effect of list type on errors was studied at the name level rather than at the physical level, using the two-letter 50\%-repetition condition. Our purpose was twofold. First, we wanted to test whether the noisy-operator theory held at the name level in addition to the physical level. Letter matches typically are detected faster than mismatches even at the name level (Bamber, 1972; Nickerson, 1972). It could well be that name matches are more often misperceived as name mismatches than vice versa, and that for this reason a "different" judgment at the name level undergoes more rechecking and thus takes longer to make. If the noisy-operator theory holds at the name level, then the same results ought to be found here as in Experiment 2, that is, more false alarms than misses on "same" lists, but more misses than false alarms on "different" lists. 
The noisy-operator theory originally was devised to explain physical-matching performance (Krueger, 1978), but it may readily be extended to the cognitive level, since verbal representations and comparisons seem no less vulnerable to distortion from internal noise than do visual representations and comparisons.

Second, we wished to eliminate the apparent motion and streaming produced by using only two letters in the two-letter 50\%-repetition condition of Experiment 2. These perceptual effects, which introduced additional cues that could have influenced performance in Experiment 2, were eliminated in Experiment 3 by eliminating stimulus repetition at the physical level. Only two letters were used, but they were shown in both their uppercase and lowercase versions, and the two letters were selected so as to have physically dissimilar uppercase and lowercase versions ( $\mathrm{Aa}, \mathrm{Dd})$. The two letters in each pair differed in case (i.e., one was uppercase, the other lowercase), as did also the two letters sharing the same position in adjacent pairs. Thus, the position of the lowercase member in the pair alternated from left to right as the successive pairs were presented. The change in case (and thus shape) at each position across pairs meant that the two letters could not readily stream perceptually, even if identical in name.

\section{Method}

The method was the same as in the two-letter 50\%-repetition condition of Experiment 2, except that $J$ and $K$ were replaced by $A$ and D. In addition, one of the two letters in each pair was converted into its lowercase version, a or d. A target "same" pair could be Aa, aA, Dd, or dD. A target "different" pair could be $A d, d A, D a$, or $d A$. The ascender of the lowercase $d$ had the same height as the uppercase $A$ and $D$, but the main portions of the lowercase a and $d$ were only about two-thirds as high and twothirds as wide as their uppercase versions. The lowercase replacement rested at the same lower level as the uppercase letter, and it was centered horizontally within the area normally occupied by the uppercase letter. The position of the lowercase letter alternated from left to right, as each successive pair in the 11-pair series was presented. In addition, its starting position, left or right, changed on each successive trial. As in the two-letter $50 \%$-repetition condition of Experiment 2, each letter pair was shown for $100 \mathrm{msec}$, with the interval between pairs left blank except for the continued presence of the fixation mark.

There were 42 subjects. Data on seven additional subjects were excluded because their errors exceeded $40 \%$.

\section{Results}

As Figure 3 shows, the same basic pattern of results was obtained as in the two-letter $50 \%$-repetition condition of Experiment 2 (see Figure 1). On RT, list type had no effect $(F<1)$, but significant effects were produced by rate of presentation $[F(3,120)=$ $3.52, \mathrm{p}<.025]$ and target presence vs. absence $[F(1,40)=37.04, p<.001]$. On errors, likewise, list type had no significant effect $[F(1,40)=2.09]$, whereas rate of presentation did $[\mathrm{F}(3,120)=169.90, \mathrm{p}<.001]$. As predicted by the noisy-operator theory, there was a significant interaction on errors between list type and target presence $[F(1,40)=82.31, p<.001]$. False

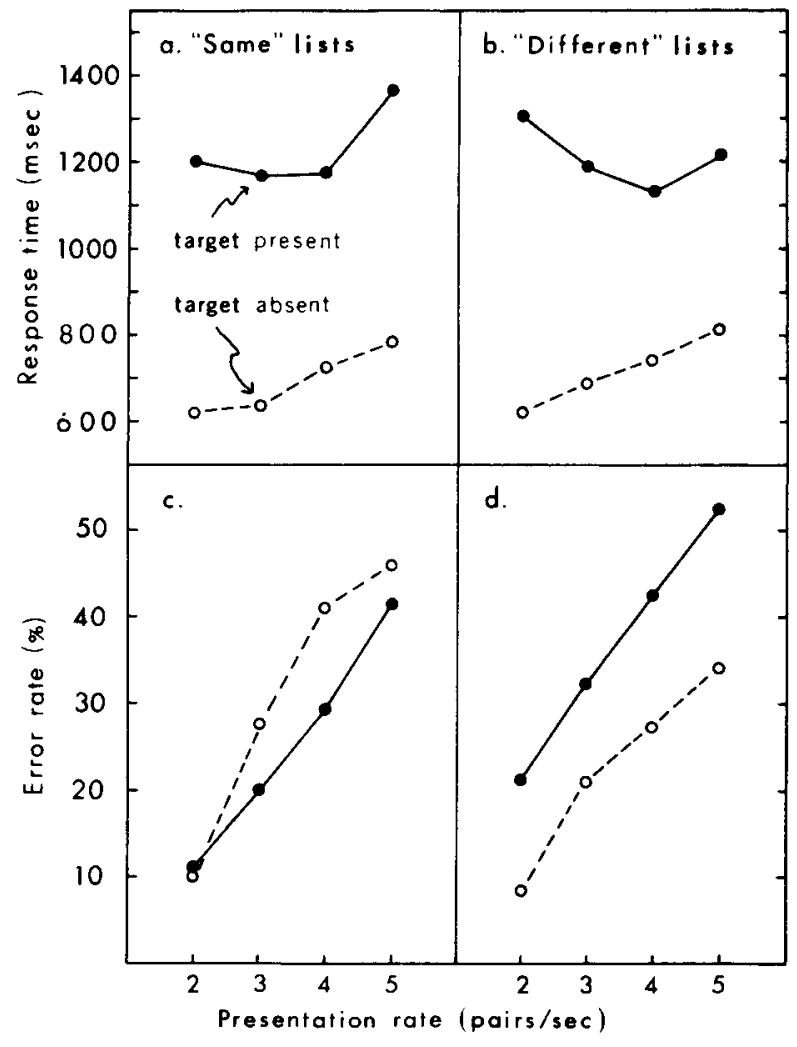

Figure 3. Experiment 3: Mean RT and percent error rate by presentation rate, list type, and target presence vs. absence. (Targetpresent RT was measured from the onset of the target pair; targetabsent RT was measured from the projected onset of a 12th pair.)

alarms outnumbered misses on "same"' lists, whereas misses outnumbered false alarms on "different" lists. This pattern was more evident at the faster presentation rates $[F(3,120)=2.93, p<.05]$. Separate analyses indicated that false alarms outnumbered misses to a marginally significant extent on "same" lists considered alone $[F(1,40)=3.69, p<.10]$, whereas misses outnumbered false alarms to a highly significant extent on "different" lists considered alone $[F(1,40)=31.84, \mathrm{p}<.001]$.

\section{Discussion}

The present results indicate that at the name level, as well as at the physical level (Experiments 1 and 2), matches are more likely to be misperceived as mismatches than vice versa (noisy-operator theory). False alarms tended to involve the detection of a nonexistent mismatch (vs. match), whereas misses tended to involve the failure to detect a match (vs. mismatch). Furthermore, by obtaining the same pattern of results as in the two-letter $50 \%$-repetition condition of Experiment 2 (see Figure 1), but without the latter condition's pattern of apparent motion and streaming, doubt is cast on the role played by apparent motion and streaming in producing the results obtained 
in Experiment 2. In Experiment 3, the identical shape never reappeared in the same position across pairs, and the characteristic perceptual pattern was for the smaller (lowercase) letter to appear to move back and forth between the left and right letter positions. Such motion presumably would not have occurred if the letters in a particular position (left, right) had fused across pairs, so the motion effect provides further indication that interruption rather than integration occurred between successive pairs.

It is conceivable that the motion effects along with the differences in size and shape between letters were sometimes taken into account, even though only the letters' names were relevant. Like internal noise, these cues may generate spurious mismatches in a sporadic or unpredictable manner, making it necessary to recheck all "different" judgments. However, the present findings indicate that subjects consistently kept their judgments at a cognitive level. If they had not, then the overwhelming number of intrapair and interpair mismatches at the perceptual or physical level would have made performance much better on the "same" lists, as shown in Figure 2 (five-letter 0\%-repetition condition in Experiment 2), whereas the actual results (see Figure 3) showed no such effect. Overall performance here, as in the twoletter 50\%-repetition condition of Experiment 2, was about the same for "same" and "different" lists.

\section{GENERAL DISCUSSION}

The error predictions of the noisy-operator theory were confirmed in whole or part by all three experiments. The typical false alarm involved detecting a nonexistent "different" pair on a "same" list, whereas the typical miss involved failing to detect a target "same" pair on a "different" list. Experiment 1 demonstrated that a search task may be used to increase the error rate, and thus to amplify the small error tendency present on individual pairs. Misses on target-present trials were much higher on "different" lists $(15 \%)$, whose target "same" pair might often be misperceived as mismatching, than on "same" lists $(10 \%)$, whose target "different" pair ought seldom to be misperceived as matching.

The data in Experiment 1 also pointed up another important aspect of the noisy-operator theory: Much or most of the distortion wrought by internal noise is removed by rechecking. Rechecking can explain why RT typically is longer on "different" pairs and why the remaining error tendency often is too small to detect. Rechecking ought to be particularly careful and complete on pairs that are singled out for special attention, such as pairs that seem at first glance to be targets, and this may explain the very low false-alarm rate $(2 \%)$ obtained in Experiment 1 and the consequent failure to find a higher false-alarm rate on "same" (vs. "different") lists, as predicted by the noisy-operator theory.

Experiments 2 and 3 used RSVP to disable the rechecking process, and thus reveal more clearly what the error effects would be like if little or no rechecking were done. False alarms appeared quite frequently in these two experiments, and, as predicted by the noisy-operator theory, false alarms outnumbered misses on "same' lists, whereas the reverse tendency held on "different" lists. Furthermore, this telltale crossover was evident not only at the physical level (Experiment 2), but also at the name level (Experiment 3). This indicates that internal noise operates in an asymmetrical manner, more often changing objective matches into mismatches than vice versa, at the cognitive level as well as at the perceptual level. The noise effect may be relatively weaker at the cognitive level, though, and no one, to our knowledge, has ever obtained a significant error effect in a singlepair name-matching task. Thus, using RSVP in Experiment 3 revealed an error tendency that might never have been found in the regular name-matching task, without running an inordinately large number of subjects and trials. All in all, the present findings indicate, once again, that errors may provide crucial information in interpreting RT data (Krueger, 1978; Krueger \& Shapiro, 1980).

The results of Experiments 1 and 2, along with those of Norman (1972), also indicate that when pairs are presented in close temporal and spatial proximity, subjects cannot avoid making and relying on extraneous interpair comparisons in addition to the relevant intrapair comparisons. Thus, the presence of many extraneous interpair mismatches in the five-letter 0\%-repetition condition of Experiment 2 prompted subjects to make many false alarms on "same" lists, that is, to report detecting a target "different" pair when none was present. When the' temporal interval is quite long, as in the regular singlepair matching task, however, then an interpair mismatch (e.g., letter nonrepetition) prompts a subject, not to respond "different," but to change the preceding response, and an interpair match prompts a subject, not to respond "same," but to keep the preceding response (Krueger $\&$ Shapiro, 1981b).

The interpair factor does not directly affect the error predictions of the noisy-operator theory. It deals with which type of list ought to do better, not with the relative change or crossover (i.e., on false alarms vs. misses) between list types. It is recommended, though, that the two-letter 50\%-repetition condition be the standard one in future work. It produces an equal number of extraneous interpair matches and mismatches, so neither list type is favored, and thus both types of lists ought to produce enough errors with RSVP to reveal quite clearly whether the telltale crossover is present, as Figures 1 and 3 indicate. 


\section{REFERENCE NOTE}

1. Sternberg, S., \& Scarborough, D. L. Parallel testing of stimuli in visual search. Paper presented at the International Symposium on Visual Information Processing and Control of Motor Activity, Sofia, Bulgaria, July 1969.

\section{REFERENCES}

Bamber, D. Reaction times and error rates for judging nominal identity of letter strings. Perception \& Psychophysics, 1972, 12, 321-326.

Fischle R, I. Detection and identification of words and letters in simulated visual search of word lists. Memory \& Cognition, $1975,3,175-182$.

Krueger, L. E. Effect of irrelevant surrounding material on speed of same-different judgment of two adjacent letters. Journal of Experimental Psychology, 1973, 98, 252-259.

Krueger, L. E. A theory of perceptual matching. Psychological Review, 1978, 85, 278-304.

Krueger, L. E., \& Shapiro, R. G. Letter detection with rapid serial visual presentation: Evidence against word superiority at feature extraction. Journal of Experimental Psychology: Human Perception and Performance, 1979, 5, 657-673.

Krueger, L. E., \& Shapiro, R. G. Why search for target absence is so slow (and careful!): The more targets there are, the more likely you are to miss one. Journal of Experimental Psychology: Human Perception and Performance, 1980, 6, 662-685.

Krueger, L. E., \& Shapiro, R. G. A reformulation of Proctor's unified theory for matching-task phenomena. Psychological Review, 1981, 88, 573-581. (a)

Krueger, L. E., \& Shapiro, R. G. Intertrial effects of samedifferent judgements. Quarterly Journal of Experimental Psychology, 1981, 33A, 241-265. (b)

Nicke RSON, R. S. Binary-classification reaction time: A review of some studies of human information-processing capabilities. Psychonomic Monograph Supplements, 1972, 4(17, Whole No. 65), 275-318.

Norman, R. Some aspects of perceptual coding. Unpublished doctoral thesis, Oxford University, 1972.

(Manuscript received September 28, 1981; revision accepted for publication February 15, 1982.) 